\title{
ESPECIES DE ACRIDOIDEA (INSECTA: ORTHOPTERA, CAELIFERA) PRIORITARIAS PARA LA CONSERVACIÓN EN URUGUAY
}

\author{
Estrellita Lorier \\ Universidad de la República. Facultad de Ciencias. Sección Entomología. Iguá 4225. Montevideo.Uruguay.
}

Autor para correspondencia: lorier@ fcien.edu.uy

\section{RESUMEN}

La preocupación por conservar la biodiversidad crece debido al fuerte impacto que las actividades productivas y la urbanización ejercen sobre ella. Para enfrentar este problema es necesario determinar las especies con mayores amenazas. La gran mayoría de las especies animales prioritarias para la conservación son vertebrados, mientras que los invertebrados están prácticamente ausentes en las listas de especies prioritarias para la conservación en Uruguay, a pesar de la gran diversidad de especies que presentan. Los acridios (Orthoptera: Acridoidea), conocidos en Uruguay como langostas del país o tucuras, son insectos herbívoros típicos de diversos ambientes del Uruguay. La principal amenaza a las que se enfrentan son los cambios en el hábitat vinculados a las actividades agrícola-ganaderas y forestales. El objetivo de este trabajo fue conformar una lista de especies de acridios prioritarias para la conservación en Uruguay. Para determinar esta lista se siguieron algunos criterios empleados por otros autores para los arácnidos del Uruguay. Se confeccionó una lista de 38 especies de acridoideos prioritarias para la conservación pertenecientes a las familias Ommexechidae (1), Romaleidae (10) y Acrididae (27). Este trabajo sienta las bases para generar acuerdos en el país y en la región en la conservación de la biodiversidad del grupo.

Palabras clave: acridios prioritarios, amenazas, Uruguay

\section{ABSTRACT}

Acridoidea species (Insecta: Orthoptera, Caelifera) priority for conservation in Uruguay. The concern to conserve biodiversity grows due to the strong impact of productive activities and urbanization exert on it. To address this problem it is necessary to determine the species with the greatest threats. The great majority of priority animal species for conservation are vertebrates, while invertebrates are practically absent in the lists of priority species for conservation in Uruguay, despite the great diversity of species they present. The grasshoppers (Orthoptera: Acridoidea), known in Uruguay as locusts of the country or tucuras, are herbivorous insects typical of different habitat of Uruguay. Changes in habitat linked to agricultural-livestock and forestry activities are the main threats. The objective of this study was to compile a list of priority acridian species for conservation in Uruguay. To create this list, some criteria used by other authors for arachnids from Uruguay were followed. A list of 38 priority acridian species for conservation belonging to the families Ommexechidae (1), Romaleidae (10) and Acrididae (27) was compiled. This work lays the foundations to generate agreements in the country and in the region in the conservation of the biodiversity of the group.

Key words: grasshoppers, priority, endangered species, Uruguay

\section{INTRODUCCIÓN}

A nivel mundial se estima que la pérdida del hábitat natural, la explotación insostenible de los recursos naturales y el cambio climático, han llevado a que la tasa de extinción de especies sea hoy mil veces mayor que las tasas naturales, y tienda a aumentar (De Vos et al., 2014). El Uruguay no escapa a esta realidad. La lista de especies prioritarias para la conservación en Uruguay resulta un insumo imprescindible para la gestión de la biodiversidad a nivel nacional y regional (Ghione et al., 2017). Existe una preocupación creciente por conservar la biodiversidad por el fuerte impacto que las actividades productivas y la urbanización ejercen sobre ella (MVOTMA y MRREE, 2016). Por lo que es necesario establecer criterios para determinar las especies con mayores amenazas y así poder establecer medidas para su conservación. La gran mayoría de las especies animales prioritarias para la conservación son vertebrados, mientras que los invertebrados están prácticamente ausentes en las listas de especies prioritarias para la conservación en Uruguay, a pesar de la gran diversidad de especies que presentan (Soutullo et al., 2013).

Las especies que conforman la siguiente lista pertenecen a la Superfamilia Acridoidea (Orthoptera), conocidas en Uruguay con el nombre común de langostas del país o tucuras. Son considerados dentro de los insectos fitófagos nativos más relevantes de nuestras praderas e integrantes destacados de las redes tróficas de este ecosistema. Algunas especies de acridoideos, suficientemente sensibles a los cambios en el hábitat, han sido utilizadas como bioindicadoras para evaluar la calidad de diferentes ecosistemas, con perspectivas a la conservación en 
áreas protegidas (Samways, 1997; Bazelet \& Samways, 2011, 2012; Hochkirch et al. 2016). La principal amenaza a la que se enfrentan son los cambios en el hábitat vinculados a las actividades agrícola-ganaderas y forestales.

Para las estrategias de conservación, es muy importante conocer la diversidad de especies ya que nuestro país forma parte de una región de América del Sur con abundantes endemismos de la fauna de acridios (Pocco, 2014; Cigliano et al., 2019). La Dirección Nacional de Medio Ambiente (DINAMA) se propuso actualizar la nómina oficial de especies de fauna silvestre e incorporar a dicha lista especies de invertebrados. Es en este marco que se elaboró y propuso la lista de acridoideos prioritarios para la conservación en Uruguay.

\section{MATERIAL Y MÉTODOS}

Para determinar las especies de acridios prioritarias para la conservación se siguieron los criterios empleados por Ghione et al. (2017) para los arácnidos del Uruguay. De los 7 criterios definidos para arácnidos se utilizaron para esta lista los siguientes:

C1: Especies endémicas de Uruguay o la región

C2: Especies poco representadas en colecciones nacionales

C3: Especies con presencia exclusiva en ambientes amenazados

C5: Especies para las cuales Uruguay sea su límite de distribución

Se resolvió emplear principalmente los criterios mencionados en esta etapa preliminar por su relevancia y su aplicabilidad en la mayoría de los casos.

Se consideraron endémicas de la región, aquellas especies con una distribución limitada a la subregión Chaqueña, dominio Chaqueño (Provincias biogeográficas del Chaco y de la Pampa) y dominio Paranaense (Provincia Paranaense), según Morrone (2014) (Fig.1 a), o provincias Pampeana, parte del Espinal, Paranaense (Sur) y Chaqueña según Cabrera y Willink (1973) (Fig. 1 b) y que comprende, el sur del estado brasileño de Río Grande Do Sul, Uruguay y las provincias argentinas de Misiones, Corrientes, Chaco,

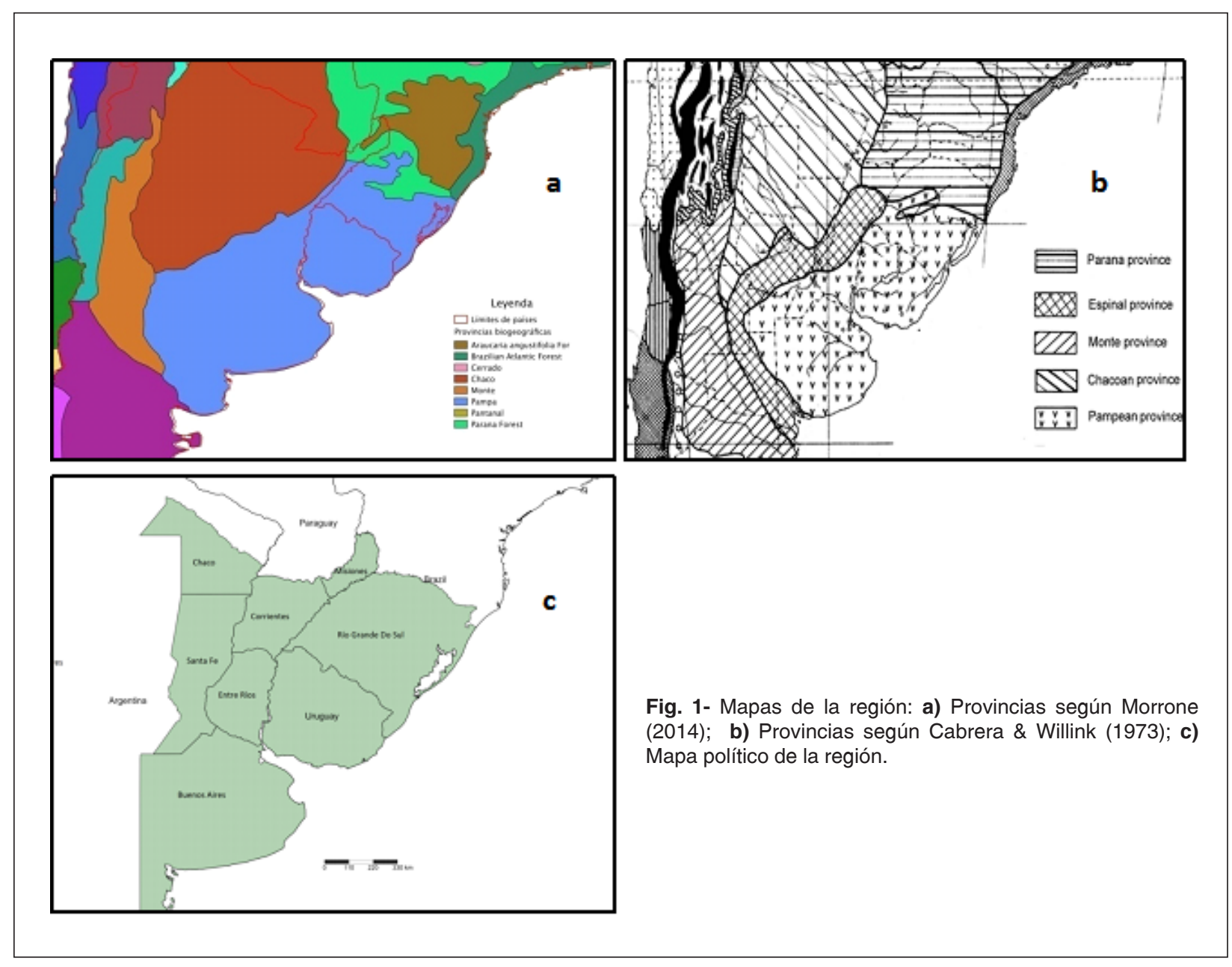

Bol. Soc. Zool. Uruguay (2ª época). 2019. Vol. 28 (2): 59-65 ISSN: 0255-4402 
Entre Ríos, Santa Fe y Buenos Aires (Fig. 1 c). Para determinar los endemismos se consultaron bases de datos generales y de la región, bibliografía específica y en algunos casos consulta a especialistas de la región.

Para la aplicación del criterio C2 se consideró el material depositado en la Colección de Entomología de la Facultad de Ciencias, Universidad de la República, Uruguay (FCE). Se contó con información digitalizada de parte del material (Ommexechidae, Romaleidae, Acrididae: Acridinae y Gomphocerinae) y se revisó el material determinado de la colección para el resto de las especies. Se fijó el criterio de poco representada cuando existían en la colección menos de 20 ejemplares.

Se realizó una revisión bibliográfica y también se consideró la aplicación de este criterio (que se indicó como $\mathrm{C} 2{ }^{*}$ ) para especies que han sido citadas para Uruguay pero no hay ejemplares en FCE. Algunos casos corresponden a especies que figuran en la bibliografía como depositadas en FCE, pero que actualmente no se conoce la localización de sus especímenes, se encuentran en préstamo o están perdidos. En otros casos los ejemplares a que refiere la cita se depositaron en otras colecciones fuera del país.

El criterio C3 se aplicó en aquellas especies que habitan ambientes acuáticos o subacuáticos y desarrollan todo su ciclo asociado a la vegetación de lugares inundados, como humedales y esteros. Se alimentan de hojas y oviponen en tallos de plantas típicas de estos ambientes.

El criterio C5 se refiere a aquellas especies de la región en las que Uruguay representa su límite de distribución conocida (aproximadamente entre los paralelos $30^{\circ}$ y $35^{\circ}$ de latitud sur y los meridianos $53^{\circ}$ y $58^{\circ}$ de longitud oeste). Para esto se consultaron las fuentes antes mencionadas en la determinación de los endemismos.

Los criterios restantes empleados por Ghione et al. (2017), a saber C4: especies con presencia exclusiva en ambientes poco frecuentes en el territorio, C6: especies con singularidad ecológica, evolutiva y comportamental, no fueron utilizados en este trabajo. Se consideró que la aplicación de estos últimos criterios requiere de información que no se dispone en forma completa en el país ni en la región para gran parte de las especies. Con respecto al criterio C7 (especies bajo amenaza a nivel nacional, debido al tráfico como mascotas) se resolvió que no es aplicable a este grupo, ya que al menos hasta el momento no se conocen casos de tráfico.

\section{RESULTADOS Y DISCUSIÓN}

Se confeccionó una lista de 38 especies de acridoideos prioritarias para la conservación en Uruguay pertenecientes a las familias Ommexechidae (1), Romaleidae (10) y Acrididae (27) (Tabla 1). Las familias más representadas en la lista fueron estas dos últimas aportando en conjunto aproximadamente un tercio del total de las especies registradas para Uruguay. (Carbonell, 2003; Carbonell et al., 2019; Cigliano et al., 2019). Las especies de Acrididae estuvieron representadas por las subfamilias Melanoplinae (12), Copiocerinae (3), Leptysminae (5), Acridinae (3) y Gomphocerinae (4). De acuerdo a la información disponible, cuatro especies son endémicas de Uruguay: Diponthus electus (Serville, 1838; Pocco, 2014), Pseudoscopas montanus (Ronderos, 1987), Aleuas albinae (Carbonell, 2008) y Borellia alejomesai (Carbonell, 1995). Se ha incluido a Diponthus electus, que fue citada también para Argentina, ya que dicha cita sería resultado de una identificación errónea. Pocco (2014) considera que correspondería a otra especie muy similar, $D$. dispar y por lo tanto la presencia de esta especie para Argentina no estaría confirmada. Hasta hace poco Aleuas uruguayensis había sido citada sólo para Uruguay (Carbonell, 2008) pero en 2014 Taffarel et al. la citaron para Argentina.

Veintiséis especies son endémicas de la región. Es de destacar el alto porcentaje de endemismos de la región (que comprende, el sur del estado brasileño de Río Grande Do Sul, Uruguay y las provincias argentinas de Misiones, Corrientes, Chaco, Entre Ríos, Santa Fe y Buenos Aires) lo que implica la necesidad de realizar acuerdos con los países vecinos en políticas de conservación para este grupo (Ronderos, 1979; Roberts \& Carbonell, 1992; Pocco, 2014; Cigliano et al., 2019).

Se incluyen 21 especies cuyo límite sur de distribución es Uruguay. Algunas de estas especies tienen una distribución limitada a la región antes mencionada y otras una distribución más amplia pero el límite sur de la misma se encuentra en Uruguay (Cigliano et al., 2019). Algunas de las especies, como Pachyossa signata, Zygoclistron trachystictum, Cylindrotettix riverae riverae, Eutryxalis filata o Borellia carinata, entre otras, han sido encontradas en Uruguay en los departamentos al Norte del Río Negro, según datos de la colección FCE y de la bibliografía de referencia. La presencia de estas especies sólo en el norte del país, tendría, entre otros factores, relación principalmente con las características del clima, el suelo y la vegetación, que determinan que el norte del Uruguay constituya el límite meridional de su distribución. Estas especies habitan ambientes de una gran diversidad que en forma prioritaria es necesario preservar. 
Tabla 1. Lista de especies de Acridoidea prioritarias para la conservación en Uruguay. La referencia de los criterios utilizados se indica en el texto. En negrita cursiva se destacan las especies endémicas del Uruguay.

\begin{tabular}{|c|c|c|c|}
\hline Familia & Subfamilia & Especie & Criterios \\
\hline Ommexechidae & Ommexechinae & Pachyossa signata Rehn 1913 & $\mathrm{C} 1, \mathrm{C} 5$ \\
\hline Romaleidae & Romaleinae & $\begin{array}{l}\text { Alcamenes brevicollis Stål } 1878 \\
\text { Alcamenes clarazianus Pictet\& Saussure } 1887 \\
\text { Alcamenes granulatus (Stål } 1875 \text { ) } \\
\text { Diponthus clarazianus Pictet\& Saussure } 1887 \\
\text { Diponthus cribratus (Serville 1838) } \\
\text { Diponthus disparGerstaecker 1873 } \\
\text { Diponthus electus (Serville 1838) } \\
\text { Staleochlora viridicata orientalis Roberts \& Carbonell } 1992 \\
\text { Zoniopoda iheringi Pictet \& Saussure } 1887 \\
\text { Zoniopoda juncorumBerg } 1887\end{array}$ & $\begin{array}{l}\mathrm{C} 1, \mathrm{C} 5, \mathrm{C} 2 \\
\mathrm{C} 1, \mathrm{C} 5 \\
\mathrm{C} 1, \mathrm{C} 2^{\star} \\
\mathrm{C} 1 \\
\mathrm{C} 1, \mathrm{C} 5, \mathrm{C} 2 \\
\mathrm{C} 1, \mathrm{C} 2 \\
\mathrm{C} 1 \\
\mathrm{C} 1, \mathrm{C} 5 \\
\mathrm{C} 5 \\
\mathrm{C} 1, \mathrm{C} 2, \mathrm{C} 5\end{array}$ \\
\hline \multirow[t]{5}{*}{ Acrididae } & Melanoplinae & $\begin{array}{l}\text { Dichroplus silveiraguidoi Libermann } 1956 \\
\text { Dichroplus patruelis (Stål1861) } \\
\text { Dichroplus robustulus (Stål 1878) } \\
\text { Dichromatos Iilloanus (Liebermann 1948) } \\
\text { Leiotettix politus Rehn 1913 } \\
\text { Neopedies orientalis Ronderos } 1991 \\
\text { Pseudoscopas campestris Ronderos } 1987 \\
\text { Pseudoscopas montanus Ronderos } 1987 \\
\text { Scotussa liebermanni Mesa \& Zolessi } 1968 \\
\text { Ronderosia piceomaculata (Carbonell 1972) } \\
\text { Ronderosia robusta (Bruner 1906) } \\
\text { Ronderosia paraguayensis (Bruner 1906) }\end{array}$ & $\begin{array}{l}\mathrm{C} 1, \mathrm{C} 5 \\
\mathrm{C} 1 \\
\mathrm{C} 1, \mathrm{C} 2 \\
\mathrm{C} 1, \mathrm{C} 5, \mathrm{C} 2 \\
\mathrm{C} 1, \mathrm{C} 5, \mathrm{C} 2 \\
\mathrm{C} 1, \mathrm{C} 5 \\
\mathrm{C} 1 \\
\mathrm{C} 1, \mathrm{C} 2^{*} \\
\mathrm{C} 1, \mathrm{C} 3 \\
\mathrm{C} 1, \mathrm{C} 5, \mathrm{C} 2 \\
\mathrm{C}, \mathrm{C}^{*} \\
\mathrm{C} 5, \mathrm{C2}^{*}\end{array}$ \\
\hline & Copiocerinae & $\begin{array}{l}\text { Aleuas albinae Carbonell } 2008 \\
\text { Aleuas uruguayensis Carbonell } 2008 \\
\text { Zygoclistron trachystictum Rehn } 1905\end{array}$ & $\begin{array}{l}\mathrm{C} 1, \mathrm{C} 2^{*} \\
\mathrm{C} 1, \mathrm{C} 2^{*} \\
\mathrm{C} 5, \mathrm{C} 2\end{array}$ \\
\hline & Leptysminae & $\begin{array}{l}\text { Cylindrotettix chacoensis Roberts } 1975 \\
\text { Cylindrotettix riverae riverae Roberts } 1975 \\
\text { Cylindrotettix santarosae Roberts } 1975 \\
\text { Haroldgrantia lignosa Carbonell, Ronderos \& Mesa } 1967 \\
\text { Cornops paraguayense (Bruner 1906) }\end{array}$ & $\begin{array}{l}\mathrm{C} 1, \mathrm{C} 5, \mathrm{C} 2 \\
\mathrm{C}, \mathrm{C2}^{*} \\
\mathrm{C}, \mathrm{C} 2^{*} \\
\mathrm{C} 1, \mathrm{C} 3, \mathrm{C} 2 \\
\mathrm{C} 5, \mathrm{C} 3, \mathrm{C} 2\end{array}$ \\
\hline & Acridinae & $\begin{array}{l}\text { Cocytotettix argentina (Bruner 1900) } \\
\text { Cocytotettix pulchripennis (Bruner 1900) } \\
\text { Eutryxalis filata (Walker 1870) }\end{array}$ & $\begin{array}{l}\mathrm{C} 1, \mathrm{C} 2 \\
\mathrm{C} 1, \mathrm{C} 2 \\
\mathrm{C} 5, \mathrm{C} 2\end{array}$ \\
\hline & Gomphocerinae & $\begin{array}{l}\text { Rhammatocerus brunneri (Giglio-Tos 1895) } \\
\text { Borellia carinata Rehn } 1906 \\
\text { Borellia alejomesai Carbonell } 1995 \\
\text { Parapellopedon instabilis (Rehn 1906) }\end{array}$ & $\begin{array}{l}\mathrm{C} 5, \mathrm{C} 2 \\
\mathrm{C} 5 \\
\mathrm{C} 1 \\
\mathrm{C} 5\end{array}$ \\
\hline
\end{tabular}

Si bien la mayoría de los acridios se asocia a ambientes de pradera algunas especies tienen preferencias más restringidas por determinados hábitats. Tal es el caso del ommexéquido Pachyossa signata, que se distribuye en un área restringida asociada a afloramientos de rocas basálticas planas a nivel del suelo, cubiertas con líquenes y musgos. Por su coloración críptica se confunden con el sustrato (Ronderos, 1979).
Son bien conocidos los hábitos acuáticos o semiacuáticos de los leptisminos, aunque algunos prefieren ambientes secos como las especies de Cylindrotettix (Roberts, 1975; Carbonell, 1981; Lhano \& Matiotti, 2008). Se incluyó en esta lista la especie Cylindrotettix santarosae que es mencionada para el Uruguay en la bibliografía (Carbonell, 2003; AssisPujol \& Pujol-Luz, 2014; Carbonell et al., 2019). Sin embargo, en la descripción original de esta especie 
(Roberts, 1975) no se menciona Uruguay en su distribución, no se encontró ninguna publicación sobre ésta que especifique la procedencia de los ejemplares examinados de Uruguay ni tampoco se hallaron datos que indiquen en qué colección estarían depositados los ejemplares.

Otras especies de pastizales, están asociadas a gramíneas densas y de alto porte y prefieren zonas bajas y húmedas, cercanas a cursos de agua como las especies de Aleuas de Copiocerinae (Carbonell, 2008). Algunas especies se las encuentra asociadas a determinadas plantas, como es el caso del romaleido Zoniopoda juncorum, cuyo nombre hace alusión a que vive sobre juncos acuáticos (Carbonell, 2007).

Otros leptisminos como Haroldgrantia lignosa y Cornops paraguayense y el melanoplino Scotussa liebermanni son especies asociadas a ambientes de humedales, zonas de bañados, terrenos bajos y anegables o inundables. Son especies estrechamente asociadas a plantas higrófilas, con oviposición endofítica y en el caso de $C$. paraguayense cumple todo su ciclo vital en la misma planta (Carbonell et al., 1967; Mesa \& Zolessi, 1968; Roberts \& Carbonell, 1980; Turk \& Aquino, 1995, 1996). Los humedales son considerados ambientes amenazados en nuestro país y en algunos casos forman parte de áreas protegidas. La protección de estos ambientes es fundamental para preservar estas especies (MVOTMA y MRREE, 2016).

Otro aspecto a considerar son los cambios en la abundancia de las especies a lo largo de los años ya que especies que eran muy abundantes hace varias décadas hoy son escasas y también ocurre lo contrario, por lo que el análisis del material de colección no permite llegar a resultados totalmente concluyentes. Además estos resultados podrían estar sesgados por esfuerzos de muestreo muy dispares en el espacio y en el tiempo.

Es de destacar que 10 de las especies de esta lista, entre ellas dos endémicas para el Uruguay, no tienen representantes en la FCE (Tabla I, $\mathrm{C}_{2}{ }^{*}$ ). Según la bibliografía consultada existen ejemplares de las mismas que están depositados en otros museos que son referencia de la fauna de acridios (Cigliano, 1997, 2007; Donato, 2000; Carbonell, 2008). Lamentablemente el holotipo de una de las especies endémicas para el país Pseudoscopas montanus estaba depositado en el Museu Nacional, Rio de Janeiro (MNRJ) (Ronderos, 1987). Poco después del devastador incendio ocurrido en dicho museo se divulgó la lista actualizada de los especímenes tipo que estaban depositados en la que figura $P$. montanus (Monné, 2018). Puesto que las colecciones entomológicas son centros de referencia en investigación sería deseable enfocar esfuerzos para procurar que aquellas especies de acridios del país que no están presentes en la colección puedan integrar su acervo.
Para evaluar el nivel de amenaza de estas especies y adjudicar una categoría de riesgo de extinción según los criterios de UICN (Unión Internacional para la Conservación de la Naturaleza) es necesario aumentar el conocimiento de las mismas, estimar el tamaño de sus poblaciones y las reducciones y fluctuaciones que experimenta, determinar la distribución espacial de la presencia y el área de ocupación de cada una y realizar análisis cuantitativos que estimen la probabilidad de extinción en cada caso (UICN, 2012). Esto permitirá realizar recomendaciones para la protección de los hábitats de las especies amenazadas. Se establece aquí un antecedente para impulsar la investigación en las especies prioritarias para la conservación en otros insectos de la fauna nativa. La protección de los hábitats de los acridios endémicos debería asegurarse con una gestión adecuada de las áreas protegidas y manteniendo un monitoreo de sus poblaciones para evaluar su amenaza.

\section{CONCLUSIONES}

Este trabajo aporta una primera aproximación al conocimiento de las especies de acridios prioritarias para la conservación en el país y en la región. Sería deseable generar un ámbito de discusión con otros especialistas de los países vecinos para acordar decisiones en cuanto a la aplicación de los criterios utilizados. Se recomienda la realización de inventarios de acridios en áreas protegidas para identificar las especies prioritarias y desarrollar estrategias adecuadas para su protección.

\section{AGRADECIMIENTOS}

Agradezco a Maria Marta Cigliano, Martina Pocco y Alba Bentos-Pereira por la colaboración brindada. Además quisiera agradecer a dos revisores anónimos que con sus sugerencias y correcciones contribuyeron a mejorar este trabajo.

\section{REFERENCIAS}

Assis-Pujol C.V. \& J.R. Pujol-Luz. 2014. Checklist de Caelifera (Acridoidea e Eumastacoidea) do Estado do Rio de Janeiro (Orthoptera). EntomoBrasilis, 7(2): 134-150.

Bazelet C.S. \& M.J. Samways. 2011. Identifying grasshopper bioindicators for habitat quality assessment of ecological networks. Ecological Indicators, 11(5):1259-1269.

Bazelet C.S. \& M.J. Samways. 2012. Grasshopper and butterfly local congruency in grassland Remnants. Journal of Insect Conservation, 16:71-85. 
Cabrera A.L. \& A. Willink.1973. Biogeografía de América Latina. Monografía 13, Serie de Biología. Organización delos Estados Americanos, Programa Regional de DesarrolloCientífico y Tecnológico, Washington D. C.120 pp.

Carbonell C.S. 1981. Aquatic biota of tropical South America. Parte 1. Arthropoda.Hurlbert S.,Rodriguez G. \& Dias Dos Santos N. (Eds.). San Diego State University, San Diego.1: 9299.

Carbonell C.S. 1995. Revision of the tribe Scyllinini, nov. (Acrididae: Gomphocerinae), with descriptions of new genera and species. Transactions of the American Entomological Society, 121: 87-152.

Carbonell C.S. 2003. Checklist of the Neotropical acridomorph species. Facultad de Ciencias, Universidad de la Republica, Montevideo, Uruguay y Museo Nacional de Río de Janeiro, Brazil. Inédita.

Carbonell C.S. 2007. The genus Zoniopoda Stål 1873 (Acridoidea, Romaleidae, Romaleinae). Journal of Orthoptera Research, 16(1): 1-33.

Carbonell C.S. 2008. The genus Aleuas Stål 1878 (Acrididae, Copiocerinae, Aleuasini). Journal of Orthoptera Research, 17(1): 1-27.

Carbonell C.S., Cigliano M.M. \& C.E. Lange. 2019. Acridomorph (Orthoptera) species from Argentina and Uruguay. Versión II [10/04/2019]. $<$ https://biodar.unlp.edu.ar/acridomorph/ >

Carbonell C. S., Ronderos R.A. \& A. Mesa. 1967. Un nuevo género y especie de Leptysminae (Orthoptera, Acrididae) de Sudamérica. La Plata. Comisión de investigación científica. Notas, (1):3-11.

Cigliano M.M. 1997. Ronderosia, a new genus of South American Melanoplinae (Orthoptera: Acrididae). Journal of Orthoptera Research, 6: 1-19.

Cigliano M.M. 2007. Review of the South American genus Eurotettix Bruner (Orthoptera, Acridoidea, Melanoplinae).Systematic Entomology, 32: 176195.

Cigliano M.M., Braun H., Eades D.C. \& D. Otte. 2019. Orthoptera Species File. Version 5.0/5.0. (10/ 04/2019) http://Orthoptera.SpeciesFile.org

De Vos J.M., Joppa L.N., Gittleman J.I., Stephens P.R. \& S.L. Pimm. 2014. Estimating the normal background rate of species extinction. Conservation Biology, 29(2): 452-462.

Donato M. 2000. Los ejemplares tipo de Orthoptera depositados en la colección del Museo de La Plata. Revista de la Sociedad Entomológica Argentina, 59(1-4): 61-84.

Ghione S., Coelho L., Costa F., García L.F., González M., Jorge C., Laborda A., Montes de Oca L., Pérez-Miles F., Postiglioni R., Simó M., ToscanoGadea C., Viera C. \& A. Aisenberg. 2017. Arácnidos prioritarios para la conservación en
Uruguay. Boletín de la Sociedad Zoológica del Uruguay (2a época), 26 (1): 1-8.

Hochkirch A., Nieto A., García Criado M., Cálix M., Braud Y., Buzzetti F.M., Chobanov D., Odé B., Presa Asensio J.J., Willemse L., Zuna-Kratky T., Barranco Vega P., Bushell M., Clemente M.E., Correas J.R., Dusoulier F., Ferreira S., Fontana P., García M.D., Heller K-G., lorgu I.S., Ivkovic S., Kati V., Kleukers R., Kristín A., Lemonnier-Darcemont M., Lemos P., Massa B., Monnerat C., Papapavlou K.P., Prunier F., Pushkar T., Roesti C., Rutschmann F., Sirin D., Skejo J., Szövényi G., Tzirkalli E., Vedenina V., Barat Domenech J., Barros F., Cordero Tapia P.J., Defaut B., Fartmann T., Gomboc S., Gutiérrez-Rodríguez J., Holusa J., Illich I., Karjalainen S., Kocárek P., Korsunovskaya O., Liana A., López H., Morin D., Olmo-Vidal J.M., Puskás G., Savitsky V., Stalling T. \& Tumbrinck J.D. 2016. European Red List of Grasshoppers, Crickets and Bush-crickets. 86 pp. Luxembourg: Publications Office of the European Union, http:// bookshop.europa.eu

IUCN Publications Services, www.iucn.org/resources/ publications

Lhano M.G. \& M.K.Matiotti da Costa. 2008. Orthoptera, Acrididae, Leptysminae, Cylindrotettix riverae riverae Roberts, 1975: New occurrence in Brazil, expanding its South American range. Check List, 4(1): 69-74.

Mesa A. \& L.C. de Zolessi. 1968. Descripción y observaciones bioecológicas sobre una nueva especie de Scotussa (Orthoptera, Acrididae). Revista de la Sociedad Uruguaya de Entomología, 7: 4-19.

Monné M.A. 2018. An updated list of the type specimens of Caelifera (Orthoptera) in the Museu Nacional, Rio de Janeiro. Zootaxa, 4462(1): 73-79.

Morrone J.J. 2014. Biogeographical regionalisation of the Neotropical region. Zootaxa, 3782(1): 1110.

MVOTMA y MRREE. 2016. Estrategia Nacional para la Conservación y Uso Sostenible de la Diversidad Biológica del Uruguay 2016 - 2020. http://www.mvotma.gub.uy/portal/

Pocco M. E. 2014. Romaleini, (Orthoptera, Acridoidea, Romaleidae) neotropicales: Biodiversidad, filogenia y biogeografía. Trabajo de Tesis doctoral. Facultad de Ciencias Naturales y Museo. Universidad Nacional de La Plata. 236 pp.

Roberts R. 1975. A Revision of the Genus Cylindrotettix including New Species (Orthoptera; Acrididae; Leptysminae). Proceedings of the Academy of Natural Sciences of Philadelphia, 127: 29-43.

Roberts H. R. \& C. S. Carbonell.1980. Concluding revision of the subfamily Leptysminae (Orthoptera, Acrididae). Proceedings of the Academy of Natural Sciences of Philadelphia, 132: $279-284$. 
Roberts H.R. \& C.S. Carbonell. 1992. Revisión of the genera Agriacris Walker 1870 and Staleochlora Nov. (Orthoptera, Romaleidae). Journal of Orthoptera Research, 1: 75-106.

Ronderos R. A. 1979. La familia Ommexechidae (Orthoptera, Acridoidea). Acrida, 8(4): 241273.

Ronderos R.A. 1987 [1985]. Revisión del género Pseudoscopas Hebard (Orthoptera, Acrididae, Melanoplinae). Revista de la Sociedad Entomológica Argentina, 44(3-4): 251-276.

Samways M. J. 1997. Conservation biology of Orthoptera. En Gangwere, S. K., Muralirangan M. C. \& Muralirangan, M. (Eds.), The Bionomics of Grasshoppers, Katydids and their Kin, 481496. Wallingford: CAB International.

Soutullo A., Clavijo C. \& J. A. Martínez-Lanfranco (Eds.). 2013. Especies prioritarias para la conservación en Uruguay. Vertebrados, moluscos continentales y plantas vasculares. SNAP/ DINAMA/MVOTMA y DICYT/ MEC, Montevideo. $222 \mathrm{pp}$.

Taffarel A., Castillo E.R.D., Acuña F.,Kraucsuk E., Marangoni F. \& D. Martí. 2014. Aleuas uruguayensis Carbonell (Orthoptera, Acrididae, Copiocerinae): novel occurrence in the
Paranaense biogeographic province. Revista de la Sociedad Entomológica Argentina, 73 (34): 191-194.

Turk S. Z. \& A. L. Aquino. 1995. Acridoideos del N.O.A VII: Estudios bioecológicos de Haroldgarantia lignosa Carbonell, Ronderos y Mesa (Acrididae Leptysminae; Tetrataeniini). Un nuevo caso de oviposición endofítica en el noroeste argentino. Acta Zoológica Lillioana, 43: 99-103.

Turk S. Z. \& A. L. Aquino. 1996. Acridoideos del NOA VIII: nuevo aporte a la bioecología y distribución del género Cornops Stal: Cornops paraguayense $(\mathrm{Br}$.$) (Acrididae: Leptysminae:$ Tetrataeniini). Acta zoológica Lilloana, 43(2):427-432.

UICN. 2012. Categorías y criterios de la Lista Roja de la UICN. Segunda edición. Gland, Suiza y Cambridge, Reino Unido: UICN VI .34 pp.

Walker F. 1870.Catalogue of the Specimens of Dermaptera Saltatoria in the Collection of the British Museum, London 4:605-809.

Fecha de Recepción: 12 de junio de 2019 Fecha de Aceptación: 27 de agosto de 2019 